\title{
Electromyographical Study of the Lower Limb Fracture During Sport Activities: Analysis from the Viewpoint of Electromyographical Kinesiology
}

\author{
Osamu Kameyama*, Ryokei Ogawa*, \\ Minayori Kumamoto** and Tsutomu Okamoto*** \\ * Department of Orthopaedic Surgery, Kansai Medical University \\ ** College of Liberal Arts, Kyoto University \\ *** College of Liberal Arts, Kansai Medical University \\ (Received for publication September 20, 1989) \\ Key Words : Fracture, EMG, Kinesiology, Biomechanics
}

\begin{abstract}
A variety of avulsion fractures have been reported in young people during sports activities. These usually occur at the insertion or origin of biarticular muscles. In the present study, an attempt was made to analyze the mechanisms of these fractures using electromyography, electrogoniometry, and motion analysis of different sporting activities. Particular consideration was given to the stress imposed on biarticular muscles. Results showed that the avulsion site reflected the "limiting factor joint" of the resultant force output. The greatest stresses were concentrated at the hip joint (origin of the rectus femoris, tensor fascia latae and sartorius) during running, and at the knee joint (insertion of the rectus femoris) during jumping. EMG analysis of the knee extensors during kicking showed that only the rectus femoris exhibited marked discharge and little or no activity was observed in vastus medialis especially in unskilled subjects. The greatest stresses were concentrated at the hip joint and the avulsion site typically occurred at the origin of the rectus femoris.
\end{abstract}

\section{Introduction}

In recent years there has been an increase in athletic injuries sustained by adolescents. In young athletes whose ligaments and muscles are elastic and strong, their own muscle power rarely causes rupture of the tendon or muscle. Instead, there is a predisposition for avulsion fractures to occur at the origin or insertion of biarticular muscles. Avulsion fractures occur at a variety of anatomical sites. In Japan, there have been reported about 100 sports related avulsion fractures of the tibial tuberosity, 70 cases for the anterior inferior iliac spine, 100 cases for the anterior superior iliac spine and 30 cases for the ischial tuberosity ${ }^{1)}$. 
Table 1

\begin{tabular}{|c|c|c|c|c|c|c|c|c|c|}
\hline Fracture Site & Side & Sex & Age & Sports Activity & Fracture Site & Side & Sex & Age & Sports Activity \\
\hline \multicolumn{5}{|l|}{ Quadriceps induced: } & \multicolumn{5}{|l|}{ Hamstring induced: } \\
\hline \multirow[t]{3}{*}{ Patellar Fracture } & $\mathrm{R}$ & $\mathrm{F}$ & 14 & Falling down & Ischial Tuberosity & $\mathrm{R}$ & $\mathrm{M}$ & 15 & Falling \\
\hline & $\mathrm{R}$ & $\mathrm{M}$ & 11 & High jump takeoff & & $\mathrm{L}$ & M & 16 & Baseball slide \\
\hline & $\mathrm{R}$ & $\mathrm{F}$ & 10 & Sprint & & & & & \\
\hline \multirow[t]{3}{*}{ Tibial Tuberosity } & $\mathrm{L}$ & $\mathrm{M}$ & 14 & Jumping (Volleyball) & \multicolumn{5}{|c|}{ Sartorius, Tensor fascia latae induced: } \\
\hline & $\mathrm{R}$ & $\mathrm{M}$ & 13 & Jumping (Basketball) & Anterior Superior & $\mathrm{R}$ & M & 14 & Sprint \\
\hline & $\mathrm{R}$ & $\mathrm{M}$ & 16 & Jumping (Basketball) & lliac Spine & $\mathrm{L}$ & M & 15 & Sprint \\
\hline Anterior Inferior & L & $\mathrm{M}$ & 13 & High jump & & $\mathrm{R}$ & M & 15 & Sprint (Soccer) \\
\hline \multirow[t]{10}{*}{ Iliac Spine } & $\mathrm{R}$ & $\mathrm{M}$ & 13 & Kicking a ball (Soccer) & & L & M & 15 & Sprint (Long jump) \\
\hline & $\mathrm{R}$ & M & 14 & Kicking a ball (Soccer) & & $\mathrm{R}$ & M & 14 & Jump (Volleyball) \\
\hline & $\mathrm{R}$ & $\mathrm{M}$ & 14 & Kicking a ball (Soccer) & & $\mathrm{R}$ & $\mathrm{M}$ & 15 & Sprint (High jump) \\
\hline & $\mathrm{L}$ & $\mathrm{M}$ & 12 & Kicking a ball (Soccer) & & $\mathrm{R}$ & M & 14 & Running \\
\hline & $\mathrm{L}$ & $\mathrm{M}$ & 16 & Jogging & & $\mathrm{R}$ & $\mathrm{M}$ & 14 & Sprint \\
\hline & $\mathrm{R}$ & $\mathrm{M}$ & 15 & Kicking a ball (Soccer) & & $\mathrm{R}$ & M & 15 & Sprint $(200 \mathrm{~m})$ \\
\hline & $\mathrm{L}$ & $M$ & 13 & Kicking a ball (Soccer) & & L & M & 15 & Sprint \\
\hline & $\mathrm{L}$ & $\mathrm{M}$ & 11 & Sprint $(100 \mathrm{~m})$ & & $\mathrm{R}$ & $\mathrm{F}$ & 13 & Sprint $(100 \mathrm{~m})$ \\
\hline & $\mathrm{R}$ & M & 14 & Kicking a ball (Soccer) & & $R$ & M & 16 & Falling down \\
\hline & $\mathrm{R}$ & $\mathrm{M}$ & 14 & Sprint & & $\mathrm{R}$ & M & 15 & Sprint $(100 \mathrm{~m})$ \\
\hline
\end{tabular}

Avulsion fracture of the tibial tuberosity has been called the "Jumping fracture" because it usually occurs while jumping in a basketball game or during high jumping. Avulsion fractures of the patella also occur at takeoff in jumping. An avulsion fracture of the anterior inferior iliac spine frequently occurs at the time of kicking the ball during rugby or football, while fractures of the ischial tuberosity and anterior superior iliac spine often occur during running ${ }^{2)}{ }^{3)}$ ). Our own observations of the type of activity being performed at the time of injury (see Table 1) are generally consistent with earlier reports associated with ball kicking ie. $70 \%$ (7 of 10) cases.

The mechanism of injury for these avulsion fractures has not been clearly identified. Epiphyseal closure usually takes place between the ages of 13-19 years, and closure of the epiphysis in the ischial tuberosity is delayed until the ages of 20-25 years. Prior to closure the bones are susceptible to fracture if large mechanical forces are applied.

In the present study we sought to further elucidate the mechanism of injury for the most common forms of these fractures. Since it is impossible to record the forces and muscle activity at the time of fracture, we analyzed isolated motions of jumping, running and kicking activities, by means of electromyography (EMG), electrogoniometry, foot switches and video analysis or cinematography. In particular, attention was focused on the activity of biarticular muscles originating or inserting at common fracture sites. The activity of the biarticular muscles was compared in skilled and unskilled young athletes with a view to identifying the circumstance and sites where the greatest tensile forces were concentrated. 


\section{Methods}

Nineteen healthy young adult male subjects participated in the study. None had any neurological or orthopaedic disorders. Ten participated in the tasks of jumping and running, and nine (of whom 3 were well-trained players and 6 untrained) in the ball kicking tasks. Electromyograms were recorded from the tibialis anterior $(\mathrm{Ta})$, the lateral gastrocnemius $(\mathrm{Gl})$, the vastus medialis $(\mathrm{Vm})$, the vastus lateralis $(\mathrm{Vl})$, the rectus femoris ( $\mathrm{Rf})$, the biceps femoris (Bf), the sartorius (Sart), the tensor fasciae latae ( $\mathrm{Tfl}$ ), the semimembranosus ( $\mathrm{Sm})$, the abductor longus ( $\mathrm{Al}$ ) and the gluteus maximus (Gmax). EMGs were recorded with conventional surface electrodes and utilizing an 18 channel electroencephalograph (San-ei Sokki Co. 1A53) in most cases, or a 4 channel elctromagnetic osillograph (San-ei Sokki Co). These systems allow a limited bandwidth of 5 to $200 \mathrm{~Hz}$ for signal recording. EMGs were obtained from the muscles of the right leg of all subjects. Video recording and high speed cinematography were used to record the motion from the side and front view. During the studies of running, foot contact events were recorded with foot switches attached to the forepart of the sole and the heel viz: "Heel-strike" the moment when the heel first strikes the ground, "Toe-on" the metatarsal heads contact with the ground, "Heel-off" the heel is lifted up, and "Toe-off" the foot is completely off the ground.

Angular changes of the knee and hip joints were recorded with electrogoniometers. The foot contact signals, the joint angular changes and the side and front view motion pictures were synchronized with the EMGs. Ten subjects performed two kinds of jumping (broad jump and vertical jump) without any preparatory running. They also performed a sprint as fast as possible. Nine subjects kicked a stationary ball using an instep kicking motion.

\section{Results}

Most of the well trained subjects performing the vertical jump showed the form of muscle activity illustrated in Fig. 1A. The muscles acting about the knee and hip joints are generally considered the main generators of the forces required for jumping ${ }^{5)}$ and have therefore been analyzed in detail. These muscles are also frequently associated with avulsion fractures.

At the vertical jump takeoff, the hip and knee joints were extended simultaneously. A strong discharge of the monoarticular Gmax and Vm commenced before extension of the hip and knee joints and continued throughout the entire extension phase. The Gmax and Vm therefore appear to be actively involved in the hip and knee extension.

The biarticular $\mathrm{Bf}$ muscle also discharged during the extension phase and contributed to hip extension. The anatomical structure of the $\mathrm{Bf}$ is such that it would also have exerted a flexion torque about the knee joint but its action here would have been opposed by contrac- 


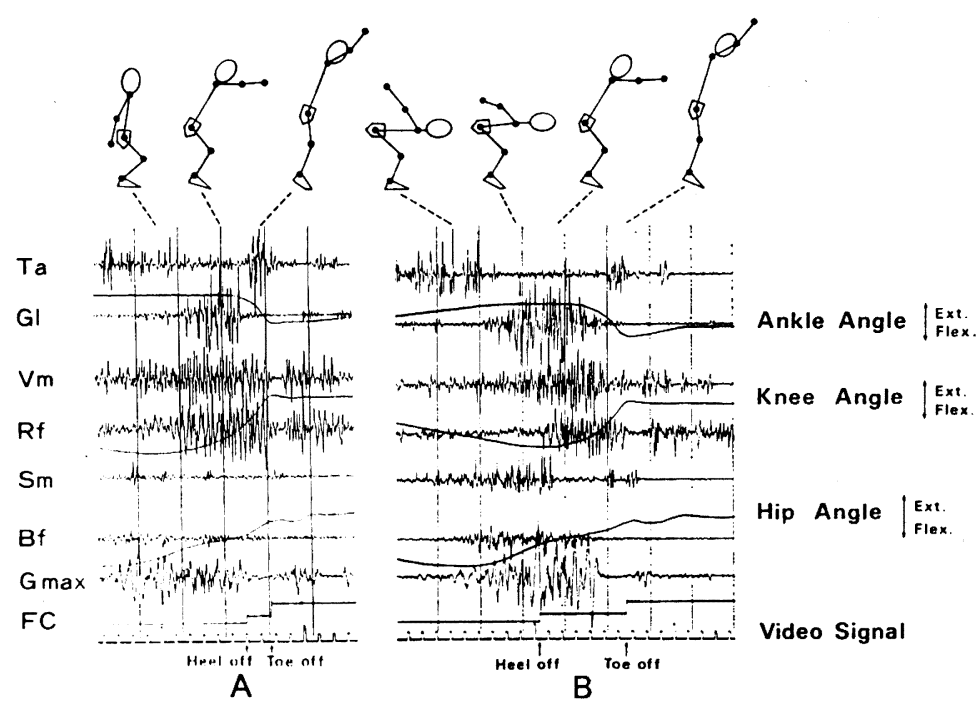

Fig. 1 Electromyograms of lower limb muscles during vertical and broad jump takeoff. $\mathrm{Ta}=$ tibialis anterior, $\mathrm{Gl}=$ gastrocnemius lateral head, $\mathrm{Vm}=$ vastus medialis, $\mathrm{Rf}=$ rectus femoris, $\mathrm{Sm}=$ semimembranosus, $\mathrm{Bf}=$ biceps femoris, $\mathrm{Gmax}=$ gluteus maximus, Vertical line represent $100 \mathrm{msec}$ intervals.

A : vertical jump, B : long jump

tion in the knee extensors eg. the Vm. The cocontraction of the $\mathrm{Bf}$ and $\mathrm{Vm}$, when the knee was fully flexed and potentially unstable, may have contributed to stabilization of the joint. The Sm was only minimally active, and its discharge also occurred when the knee was fully flexed. It, too, may have contributed to stabilization of the knee joint. The Rf showed some activity throughout the crouch, and increased its firing during the hip and knee extension. Its activity was largely reciprocal to that of the two biarticular hip extensors Bf and Sm.

Kumamoto $^{6)}$ has reported that in tasks involving simultaneous knee and hip extension movement, the resultant force output at the foot does not represent the summation of force outputs from both the knee and hip, but reflects the weaker of the forces (or joint torques). The joint with the weakest force (or torque) output thus constitutes the limiting factor. Yamashita ${ }^{7)}$ and Kumamoto ${ }^{8}$ ) further reported that the reciprocal electrical discharge patterns of antagonistic biarticular muscles (Rf and Bf) were completely reversed depending on whether the resultant force output was limited by either the knee joint force, or the hip joint force. The joint that constituted limiting factor, was determined by the proximity of the line of action of the resultant force vector. Thus, the $\mathrm{Rf}$ showed marked discharge when the line of action of the resultant force passed close to the hip joint, and the knee joint constituted the limiting joint. Conversely, the Bf was predominantly active when the 
line of action passed close to the knee joint and in this case the hip joint was the limiting joint. In the case of the vertical jump, therefore, with the body in an erect position, the line of action of the resultant force vector would have passed closer to the hip than to the knee. This would have necessitated substantial force development in the Rf, but little in the Bf. This method of identification of the limiting factor in the resultant force output may elucidate the mechanism of some of the avulsion fractures, since avulsion fractures of the tibial tuberosity are frequently seen $(65 \%)$ in the vertical jump, and avulsions of the ischial tuberosity are infrequently seen. ${ }^{11}$

Untrained subjects did not exhibit the same degree of reciprocation between the activity of the Rf and Bf. Their neuromuscular coordination was therefore less efficient and would have limited the magnitude of the vertical thrust (resultant vertical force) that the untrained subjects could generate during hip and knee extension. Their cocontraction of forcefully contracting antagonistic muscles may especially predispose untrained subjects to avulsion fractures in the origins or insertions of the biarticular muscles. (In order to maintain the same net joint torque, the quadriceps force would need to be greater than when the antagonist hamstring muscle is inactive.)

In young people whose performance of jumping skill develops between 14 and 16 years of age, the epiphyseal growth plates frequently are not strong enough to sustain the traction force imposed by the muscular activity. When a young athlete jumps vertically, the large force of the quadriceps contraction acts on the tibial tuberosity. In an untrained jumper, avulsion of the tibial tuberosity seems to occur more easily perhaps because the inappropriate force of the cocontracting antagonists takes place simultaneously. The additional force required in the quadriceps to sustain the net torque output may lead to separation of the tibial tuberosity from the tibia, at the epiphyseal plate of the tuberosity.

Fig. 1-B shows representative EMGs during the long jump. There was no difference in the ankle joint movement between the vertical and long jump. The hip joint displacement changed from flexion to extension prior to the time of the heel-off. A marked discharge of the Gmax was obvious during this hip extension phase. In the initial stage of the hip extension, when the knee joint was still flexed, the $\mathrm{Sm}$ and $\mathrm{Bf}$, the biarticular muscles, demonstrated substantial activity. The knee joint began to extend between the heel-off and toe-off. The gradually increased discharge of the Vm appeared to function to regulate the knee joint which was passively flexed, supporting the upper body load, and then assisted in the active extension of the knee joint. The Rf muscle output, which commenced about 200 msec before the toe-off, contributed to the extension of the knee joint in the same fashion as the Vm. However, the Rf was not activated during the period when the hamstrings were 
most active. The Rf exhibited greater activity for about $150 \mathrm{msec}$ prior to the toe-off. At that time there was a reciprocal depression of activity in the hamstring muscles. In the broad jump, the limiting factor of the resultant force output appears to be the hip joint, in the initial stage when the knee joint is flexed, and in the knee joint in the later stage. This may explain the low incidence $(\sim 6 \%)$ of avulsions of the tibial tuberosity sustained during the broad jump. ${ }^{1)}$

In the case of unskilled subjects, a clear reciprocal discharge pattern of the biarticular muscles was rarely observed during the takeoff. Cocontraction of the $\mathrm{Sm}, \mathrm{Bf}$, and $\mathrm{Rf}$ was usually seen not only during the broad jump but also in the vertical jump. The marked activity of the hamstrings which appeared with the Rf prior to the toe-off might have generated the large force necessary to pull the lower extremity backward.

Fig. 2 shows a representative pattern of EMGs during sprinting. In the latter half of the swing phase, the hip and knee joint extend simultaneously prior to heel strike. Prior to heel-strike the Vm and Gmax showed substantial activity and might function to extend the hip and knee. The knee joint showed a small flexion at the heel-strike which probably represents a passive flexion and contributes to absorbing the shock of the heel-strike. During the stance phase, marked discharge was observed in the $\mathrm{Bf}$ but the Rf showed little activity. This suggests that the resultant force output of the sprint might be limited by the hip extension force. If so, the stress of driving forward is concentrated in the hip extensors.

At the end of the stance phase, the Tfl and Sart began to show activity. These two muscles, which are biarticular muscles, acting as flexors for both the knee and hip joints, appear to contribute to knee flexion and hip flexion during the early part of the swing phase. The Rf began to show activity slightly after the Tfl and Sart appeared to contribute to hip flexion.

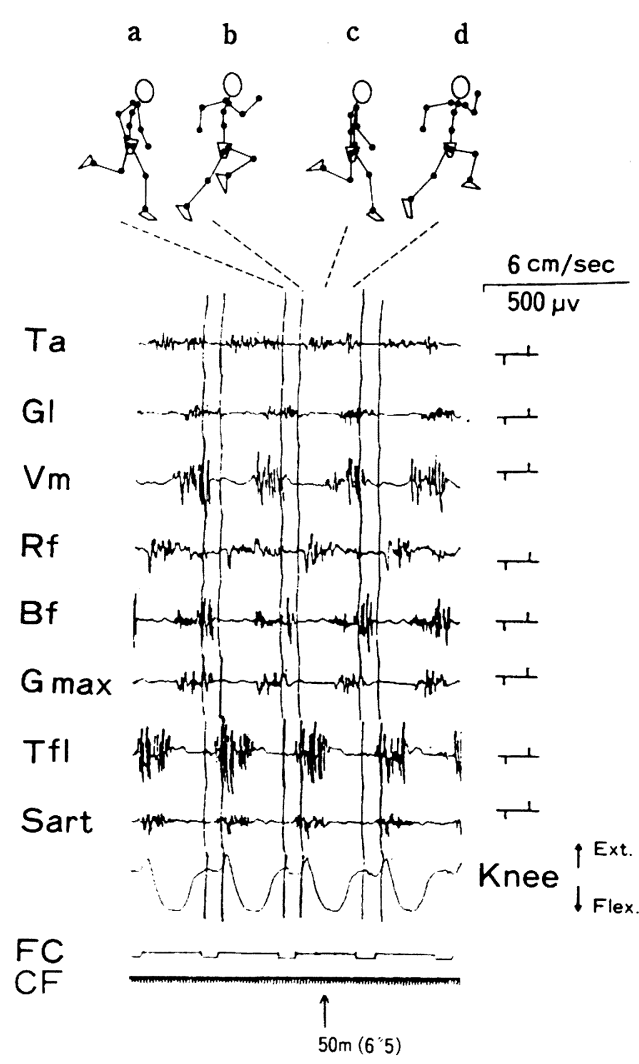

Fig. 2 Electromyograms of lower limb muscles during sprinting. FC (foot contact) shows the heel-strike (a), toe-off (b), early swing (c) and late swing (d). $\mathrm{Tfl}=$ tensor fascia latae, $\mathrm{CF}=$ cinematography frames. 
Therefore, the origin of the Tfl and Sart might be a point of mechanical weakness in the latter half of the stance phase, during pushing off, because these muscles began to contract strongly while the leg was still forcefully extending. ${ }^{\text {9) }}$

Although avulsion of the ischial tuberosity often occurs during sprinting, it usually happens with an accidental trigger such as slipping when the coordination of the muscles is disturbed 10). That is, the separation of the ischial tuberosity might be inferred to take place during conditions when large external passive force or improper muscle coordination functions to distend the hamstring while it contracts strongly in the stance phase.

Fig. 3 shows the EMG pattern of a skilled and unskilled kicker while kicking the soccer ball. At the point of impact the knee was extended and the hip was flexed. Following that, the hip joint continued to flex and the leg swung through. The Rf, the knee extensor and hip flexor invariably showed high levels of activity during the leg swing and ball impact, both in skilled and unskilled kickers. During this period the Vm, the monoarticular knee extensor, revealed a large burst of activity in all skilled subjects, but not in the unskilled kickers. This discharge pattern showed not only the inefficient muscular activities of the unskilled subject but also that the stress associated with ball kicking was concentrated in the Rf.

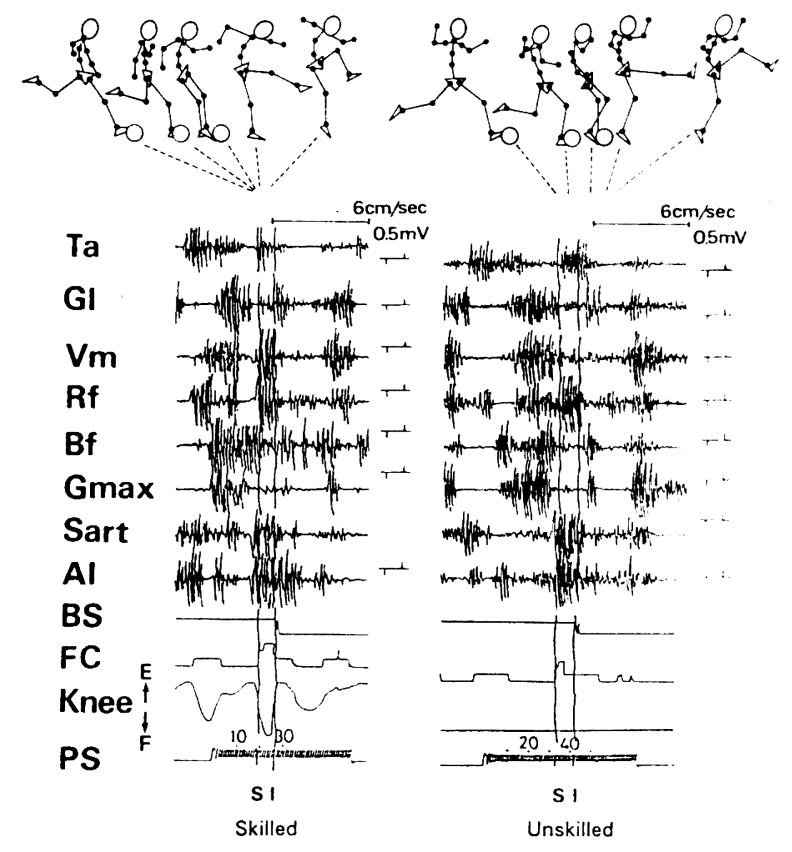

Fig. 3 Electromyograms of lower limb muscles of skilled and unskilled athletes while kicking the soccer ball.

$\mathrm{Al}=$ adductor longus, $\mathrm{BS}=$ ball strike, $\mathrm{S}=$ heel strike of the opposite foot, I=impact on the ball. 
In young children, the pelvic origin of th $\mathrm{Rf}$ is constituted with epiphyseal cartilage and it is mechanically weak. Therefore if excessive stresses are imposed on the Rf, eg. during kicking, an avulsion fracture would be likely to occur at the anterior inferior iliac spine. During the follow through from kicking, the leg was swung up and there was generally little activity in the Gmax and Bf, although a few subjects showed some activity in the Bf at this time. Takagi ${ }^{11)}$ described a strong discharge of the Bf during instep kicking in skilled players that seemed to be induced by the specific kicking method, that is, stabilizing the hip joint before impact and "chipping" the ball ie. without the leg swinging up. Some variations of the activity patterns of the $\mathrm{Bf}$ in the present study might be attributed to the fact that some subjects kicked the ball in this manner. In these cases the strong discharge of the Bf might serve as a counter traction against the contracted $\mathrm{Rf}$ at the hip joint. In young athletes this method of kicking might therefore lead to avulsion fracture of the anterior interior iliac spine.

\section{Discussion}

When one considers the mechanism of avulsion fractures from indirect forces, as in forceful muscular contraction, there is necessity for analysis from various perspectives. In the present study, the activities of the biarticular muscles were examined in jumping and running and an attempt was made to establish which joint, knee or hip joint was the limiting factor of the resultant force output, that is, at which joint the muscular stresses were concentrated. We conclude that the site of avulsion at the origin or insertion of a specific muscle depends on the concentration of muscular stresses. In the knee joint, it was unclear from our analysis whether the tibial tuberosity or patella would be at greater risk. The answer to this may be related to the knee joint angle at the time of the greatest muscular stress. The biarticular muscle functions during ball kicking were different from those in jumping and running. The knee extensor forces were concentrated within the Rf because the other knee extensors were not active. The knee extensors, except for the Rf, were not activated in unskilled players, so the greatest stress seemed to be concentrated in the origin of the Rf, at the hip joint. This is consistent with the observed site of avulsion injuries sustained during these activities.

Young athletes appear to be at increased risk of incurring avulsion fractures at the origin or insertion of biarticular muscles because of their lack of well developed neuromuscular patterns for execution of the sports skills (leading to muscular cocontractions) and the relatively late closure of the epiphyseal plates. They become particularly prone to avulsion fractures when they participate in strenuous muscular activities that involve large muscular 
forces. We recommend that careful consideration should be given to the development of training methods that allow for development of the requisite pattern of muscular coordination and strength, prior to allowing childeren to participate in extremely strenuous activity.

\section{Acknowledgments}

We would like to thank Doctor Keith C. Hayes, University of Western Ontario, London, Canada, for his critical reading of the manuscript. This work was supported by the Grant-in Aid for Co-operation Research, 62771105, the Ministry of Education, Science and Culture, Japan.

\section{References}

1) Kameyama, O., Oka, H., Hashimoto, F., Nakamura, K., Hatano, I., Okamoto, T. and Kumamoto, M. : The avulsion fracture of the tibial tuberosity as a result of violent muscle contracture. Biomechanics 8-A, 157 162, 1983.

2) Kobayashi, A. : Clinical study of the avulsion fracture of the anterior superior iliac spine. Orthop. Surg., 37, 1853 1861, 1986.

3) Watson-Jones, R. : Fractures and Joints Injuries 6th Ed. 853 855, Churchill Livingstone, 1982.

4) Weitzwe, I. : Fracture of the anterior superior spine of the ilium in one case. Am. J. Roentgenol., 33, 39 40, 1935.

5) Hubley, C.L. and Wells, R. O. : A work-energy approach to determine individual joint contributions to vertical jump performance. Eur. J. Appl. Physiol., 50, 247 254, 1983.

6) Kumamoto, M., Oka, H., Kameyama, O., Okamoto, T., Yoshizawa, M. and Horn, L. : Possible existence of antagonistic inhibition in double joint leg muscles during normal gait cycle. Biomechanics 7-B, 157 172, 1979.

7) Yamashita, N. : The mechanism of generation and transmission of forces in leg extension. J. Human Ergol., 4, 43〜52, 1975.

8 ) Kumamoto, M. and Takagi, K. : Dynamic and neurophysiological features in serial multijoints movements In : Takagi, K. and M. Kumamoto (Eds.), Regulation of Physical Activity, 207 299, Kyorin Shoin Ltd., Tokyo, 1980.

9) Mann, R. and Sprague, P. : A kinetic analysis of the ground leg during sprint running. Res. Q. Exercise \& Sports ; 334 348, 1980.

10) Hamada, G. and Rinda, A. : Ischial apophysiolysis. Clin. Orthop., 31, 117 130, 1963.

11) Takagi, K. : EMG study of ball kicking. Sports, 55, 79 83, 1961. 